\begin{tabular}{|c|l|}
\hline Title & Severity of A D HD symptoms and efficiency of attentional resource all location \\
\hline Author(s) & Sawaki, Risa; Katay ama, Jun'ichi \\
\hline Citation & $\begin{array}{l}\text { Neuroscience Letters, 407(1), 86-90 } \\
\text { https://doi.org/10.1016/.neulet.2006.08.006 }\end{array}$ \\
\hline Issue Date & $2006-10$-16 \\
\hline Doc URL & http://hdl.handle.net/2115/15819 \\
\hline Type & article (author version) \\
\hline File Information & NL407-1.pdf \\
\hline
\end{tabular}

Instructions for use 
Neuroscience Letters, 2006, 407, 86-90.

\title{
Severity of AD/HD symptoms and efficiency of attentional resource allocation
}

\author{
Risa SAWAKI ${ }^{1,2}$ \& Jun'ichi KATAYAMA ${ }^{1}$ \\ ${ }^{1}$ Graduate School of Education, Hokkaido University, Japan \\ ${ }^{2}$ Research Fellow of the Japan Society for the Promotion of Science, Japan
}

Corresponding author:

Risa SAWAKI

Graduate School of Education, Hokkaido University Kita-11 Nishi-7, Kita-ku, Sapporo 060-0811, JAPAN

Tel \& Fax: +81-11-706-3113

E-mail: sawaki@edu.hokudai.ac.jp 


\section{Abstract:}

This study investigated the mechanism that underlies the inefficient allocation of attentional resources in $\mathrm{AD} / \mathrm{HD}$. The P300 event-related brain potential (ERP) was elicited from 24 healthy adults using a visual three-stimulus oddball paradigm (standard, 70\%; target, 15\%; non-target, 15\%) and the degree of their $\mathrm{AD} / \mathrm{HD}$ symptoms was assessed by using AD/HD symptom scales. Target stimulus was a circle and standard stimulus was an "X". Two task conditions were defined according to the non-target stimulus type (typical or novel): a triangle for the typical condition and colored non-repetitive novel stimuli for the novel condition. In both conditions, target and non-target elicited P300s. A ratio of non-target P300 to target P300 amplitude was used to assess the efficiency of attentional resource allocation; low ratio indicates the efficient allocation of attentional resource. The correlation analysis revealed a strong positive correlation between the $\mathrm{AD} / \mathrm{HD}$ symptom score and the $\mathrm{P} 300$ amplitude ratio in the typical condition $(r=.80)$, while only a weak positive correlation was observed in the novel condition $(r=.23)$. The present study found that the commonality of task-relevant and task-irrelevant information, rather than the stimulus novelty of task-irrelevant information, induces the inefficient allocation of attentional resources in $\mathrm{AD} / \mathrm{HD}$. 


\section{Key words:}

$\mathrm{AD} / \mathrm{HD}$; attentional resource allocation; P300; target; non-target; three-stimulus oddball paradigm 


\section{Introduction}

Attentional resource allocation is crucial for recognizing the significance of various events which occur in the external environment. However, since attentional capacity is not infinite, an efficient allocation of limited attentional resources is important to achieve a goal-directed behavior. If task-irrelevant information consumes valuable attentional resources, task-relevant information may not be processed sufficiently. Thus, the inefficient allocation of attentional resources could lead to a failure of goal-directed behavior.

Attention-Deficit/Hyperactivity Disorder $(\mathrm{AD} / \mathrm{HD})$ is one of the most common childhood disabilities and is characterized by developmentally inappropriate symptoms of inattention, impulsivity and hyperactivity [1, 3], which persist into adulthood [21]. Extensive studies have shown that children and adults with AD/HD perform worse than normal controls on broad range of cognitive and attentional tasks, such as the go/no-go task, stop-signal task, and oddball task. They often show a longer response time, a lower hit rate, and a higher false positive rate [e.g., 11, 22, 27], all of which indicate that the cognitive mechanism of $\mathrm{AD} / \mathrm{HD}$ may be associated with the inefficient allocation of attentional resources. However, the mechanism that underlies the inefficient allocation of attentional resources in $\mathrm{AD} / \mathrm{HD}$ has not yet been elucidated.

It has been known that attentional resources could be allocated not only for 
task-relevant but also for task-irrelevant information, which often interferes with the task-relevant processing and tends to occur to infrequent task-irrelevant information [e.g., 8]. The inefficient allocation of attentional resources could be due to several factors. One factor is the commonality of task-relevant and task-irrelevant information, which controls the allocation of attentional resources for not only task-relevant but also task-irrelevant information $[2,31]$, and thus task-irrelevant information would deplete attentional resources. Another factor is the stimulus novelty of task-irrelevant information, which provides a strong draw for attention, and thus novel information would be allocated attentional resources even if it is task-irrelevant [9]. It has not yet been elucidated which factor induces the inefficient allocation of attentional resources in $\mathrm{AD} / \mathrm{HD}$. The main purpose of the present study was to clarify this issue.

We investigated the correlation between the severity of $\mathrm{AD} / \mathrm{HD}$ symptoms in healthy young adults and the efficiency of attentional resource allocation in two different conditions of an infrequent target detection task; one in which the infrequent non-target is a typical stimulus like the target and frequent standard stimuli, and another in which the non-target is a highly novel stimulus. Wodushek and Neumann (2003) demonstrated that a study for non-diagnosed adults could provide rich information regarding the cognitive mechanism of $\mathrm{AD} / \mathrm{HD}$ by using $\mathrm{AD} / \mathrm{HD}$ symptom scale [33]. Since most previous studies have been based on a between-groups design (e.g., AD/HD vs. normal control, or low vs. high $\mathrm{AD} / \mathrm{HD}$ symptom group), there are no 
studies that directly examined the correlation between the AD/HD symptom score in non-diagnosed adults and their neural activities regarding the attentional resource allocation. The present study examined this correlation directly.

To elucidate the neural mechanism of attentional resource allocation, several studies have used the event-related brain potential (ERP). One of the most widely studied ERP components is $\mathrm{P} 300$ or P3 [25, 28, 29]. This component is usually obtained with the so-called "oddball paradigm", which is based on the random occurrence of infrequent stimuli that are embedded in a train of frequent standard stimuli. In a classical oddball paradigm, or "two-stimulus" oddball paradigm, a participant has to respond to each infrequent target stimulus by pressing a button or by silent counting. Target stimuli elicit P300, which is a large positive-going potential that has a maximum amplitude over parietal scalp electrode sites with a peak latency of about 300-600 ms, depending on the stimulus modality and task difficulty [14, 23]. It has been considered that the amplitude of P300 reflects the allocation of attentional resources [18, 32], and its peak latency reflects the time required for stimulus classification $[19,20]$.

One variant of the oddball paradigm, the "three-stimulus" oddball paradigm, presents the participant with an additional infrequent non-target stimulus that is inserted into the sequence of target and standard stimuli. An infrequent non-target also elicits a P300 component. In general, non-target P300 has a more anterior distribution 
than the parietally distributed target P300, although the properties of the non-target P300 component vary with experimental conditions $[6,15,16,17]$.

As mentioned above, the P300 component could be elicited by both target and non-target stimuli, and its amplitude reflects the allocation of attentional resources. If the efficient allocation of attentional resources is defined as a maximum allocation for task-relevant information and a minimum allocation for task-irrelevant information, it should be reflected by a larger P300 amplitude for target stimuli and a smaller P300 amplitude for non-target stimuli. One problem arising here is that there are individual differences in the absolute value of the P300 amplitude itself that are due to non-cognitive factors (e.g., anatomical factors, biological factors, and background EEG factors $[10,12,26])$. This issue would not be a critical problem in a within-participant design or between-groups design comparing diagnostic and control groups. However, when a correlational approach is employed, this issue would be critical. Therefore, a ratio of non-target $\mathrm{P} 300$ to target $\mathrm{P} 300$ amplitude would be a better measure to assess the efficiency of attentional resource allocation properly because the individual differences in the absolute value of the P300 amplitude could be canceled out; low ratio indicates the efficient allocation of attentional resource.

\section{Methods}

Twenty-four healthy young adults (10 men and 14 women, 23 right-handed and 1 
ambidextrous) with normal or corrected-to-normal visual acuity and normal color vision served as participants (range $=20-32$ years, $M=23.3$ years, $S D=2.8$ years). All participants reported being free of neurological or psychiatric disorders and provided written, informed consent.

Two kinds of Japanese-translated AD/HD symptom scales were used to assess the degree of AD/HD symptoms: the Wender Utah Rating Scale (WURS) [30] and the Current Symptoms Scale [4]. Since Ward et al. (1993) [30] reported that 25 WURS items (of the total 61) were particularly good for discriminating between adults with and without $\mathrm{AD} / \mathrm{HD}$, the present study used these 25 items (score range: $0-4$ each) in WURS. The Current Symptoms Scale contains 18 symptom items (score range: 0-3 each) for $\mathrm{AD} / \mathrm{HD}$ from DSM-IV in the form of a self-report rating scale. Because both scores showed a similar tendency with respect to the P300 amplitude ratio, the summated score of the WURS and Current Symptoms Scale was used to give individual $\mathrm{AD} / \mathrm{HD}$ symptom scores (total possible score range: 0 - 154).

Stimuli were presented on a computer monitor, placed $1 \mathrm{~m}$ in front of the participant, in a random series once every 2 s, with a $200 \mathrm{~ms}$ duration. In each experimental condition, 300 stimuli were presented, consisting of standard, target, and non-target with probabilities of $.70, .15$, and .15 , respectively. Target stimulus was a circle and standard stimulus was an " $\mathrm{X}$ " in both conditions. Two task conditions were defined according to the non-target stimulus type (typical or novel): a triangle for the 
typical condition and colored non-repetitive novel stimuli for the novel condition. All stimuli were $3.2 \mathrm{~cm}$ high $\times 3.2 \mathrm{~cm}$ wide. The participants were instructed to respond to the target stimuli by pushing a button with the right thumb as quickly as possible. Each experimental condition was divided into three experimental blocks and each block lasted about $3.4 \mathrm{~min}$. The order of the two conditions was randomized across participants.

Electroencephalographic (EEG) activity was recorded from 4 midline electrodes at frontal $(\mathrm{Fz})$, central $(\mathrm{Cz})$, parietal $(\mathrm{Pz})$, and occipital $(\mathrm{Oz})$ sites according to the 10-20 System. Both earlobes were used as the reference, and the forehead was used as a ground. Voltage changes caused by eye movements and blinks were monitored with two electrooculogram (EOG) recordings from bipolar electrodes placed at the outer canthi of each eye, and above and below the right eye. Impedance was kept at $10 \mathrm{k} \Omega$ or less. The EEG and EOG signals were amplified with a bandpass of $0.05-30 \mathrm{~Hz}$ and digitized at $250 \mathrm{~Hz}$. Averaging epochs were $900 \mathrm{~ms}$, beginning $100 \mathrm{~ms}$ before stimulus onset. Waveforms were averaged off-line, such that trials with a response error or those in which the EEG or EOG exceeded $\pm 100 \mu \mathrm{V}$ were rejected automatically.

The P300 component was defined as the largest positive-going peak between 300 and $700 \mathrm{~ms}$ after stimulus onset. Peak latencies for target and non-target were measured from the time of stimulus onset. Peak amplitudes for target and non-target 
were measured relative to the prestimulus baseline at the $\mathrm{Pz}$ (target) and $\mathrm{Cz}$ (non-target) peak latency points. The non-target $(\mathrm{Cz}) /$ target $(\mathrm{Pz}) \mathrm{P} 300$ amplitude ratio was calculated individually, and a correlation analysis was used to investigate the relationship between the degree of $\mathrm{AD} / \mathrm{HD}$ symptoms and the non-target/target $\mathrm{P} 300$ amplitude ratio.

\section{Results}

Table 1 shows the mean reaction time, hit rate, and false positive rate. Behavioral performance was similar between the two conditions, and one-way ANOVAs revealed no significant differences.

Fig. 1 shows the grand averaged ERPs from the four midline electrodes for the standard, target, and non-target stimuli in each condition. Targets elicited a $\mathrm{Pz}$ maximum P300 component and non-targets elicited a Cz maximum P300 component. The amplitude data from the $\mathrm{Pz}$ (target) and $\mathrm{Cz}$ (non-target) electrodes were assessed with a two-factor ( 2 conditions $\times 2$ stimulus categories) ANOVA. This analysis revealed the main effect of stimulus category, which indicates that the P300 amplitude for target was greater than that for non-target, $F(1,23)=89.9, p<.001$. No significant main effect of condition or its interaction was observed. The same analysis for the latency found neither main effects nor interaction.

Fig. 2 shows scattergrams and Pearson's $r$ correlation coefficients to illustrate 
the relationship between the degree of $\mathrm{AD} / \mathrm{HD}$ symptoms and the non-target/target P300 amplitude ratio. This correlation analysis revealed a strong positive correlation between the $\mathrm{AD} / \mathrm{HD}$ symptom score and the non-target/target $\mathrm{P} 300$ amplitude ratio in the typical condition, $r(22)=.80, p=.001$, while only a weakly positive correlation was observed in the novel condition, $r(22)=.24, p>.2$. There were weak or no correlations between the degree of $\mathrm{AD} / \mathrm{HD}$ symptoms and the absolute values of the P300 amplitude (typical condition: target $r(22)=-.41$, non-target $r(22)=.09$; novel condition: target $r(22)=-.25$, non-target $r(22)=-.05)$. In addition, there were no significant correlations between the $\mathrm{AD} / \mathrm{HD}$ symptom score and the P300 latency or behavioral performance.

\section{Discussion}

In both conditions, target and non-target stimuli elicited P300s that had similar latencies. The amplitudes for the target were larger than those for the non-target stimuli. These results are consistent with previous reports using a three-stimulus oddball paradigm [24].

The non-target/target P300 amplitude ratio reflects the efficiency of attentional resource allocation, and effective allocation is reflected by a reduction in this ratio. The present study found that the $\mathrm{AD} / \mathrm{HD}$ symptom score in healthy adults is strongly and positively correlated with the non-target/target P300 amplitude ratio in the 
typical condition. This finding suggests that individuals with a higher degree of $\mathrm{AD} / \mathrm{HD}$ symptoms tend to allocate their attentional resource ineffectively, at least when a non-target stimulus has attributes similar to those of a target stimulus. In contrast, there was only a weak positive correlation in the novel condition. These results suggest that the commonality of task-relevant and task-irrelevant information, rather than the stimulus novelty of task-irrelevant information, enhances the association between the severity of AD/HD symptoms and the efficiency of attentional resource allocation. Therefore, it is reasonable to conclude that the commonality of task-relevant and task-irrelevant information induces the inefficient allocation of attentional resources in $\mathrm{AD} / \mathrm{HD}$.

In the present study, the $\mathrm{AD} / \mathrm{HD}$ symptoms are rated in a non-patient sample, resulting in a wide range of symptom scores (score range in the present study was 20-115 within the possible range of $0-154$ ), which makes correlation analysis useful. The $\mathrm{AD} / \mathrm{HD}$ symptom score in $\mathrm{AD} / \mathrm{HD}$ patients would be similar or higher than the high scores in the present study. Thus, similar association of AD/HD symptoms with the efficiency of attentional resource allocation would be expected in $\mathrm{AD} / \mathrm{HD}$ patients, although further research is required.

The present study showed the electrophysiological differences in the absence of performance differences. One possibility is that the absolute value of the reaction time, as well as the P300 amplitude, is ineffective, in particular with non-patient 
sample and correlational approach. Furthermore, a similar result pattern has also been found even in the diagnosed $\mathrm{AD} / \mathrm{HD}$ patient studies (e.g., [13]), which indicates that the electrophysiological marker could be a more sensitive measure in some cases.

This is the first report that demonstrates the strong correlation between the degree of $\mathrm{AD} / \mathrm{HD}$ symptoms in non-diagnosed adults and their neural activities regarding the attentional resource allocation. This finding provides important evidence for the cognitive mechanism of $\mathrm{AD} / \mathrm{HD}$ which has been discussed in many neuroscience studies [e.g., 5, 7]. Furthermore, it is reasonable to suppose that the three-stimulus oddball paradigm using typical stimuli can be used to assess the attentional function of children and adults with $\mathrm{AD} / \mathrm{HD}$ and candidates for $\mathrm{AD} / \mathrm{HD}$. 


\section{References}

1. American Psychiatric Association, Diagnostic and Statistical Manual of Mental Disorders, 4th ed, American Psychiatric Association, Washington, DC, 1994.

2. A. Azizian, A.L. Freitas, T.D. Watson, N.K. Squires, Electrophysiological correlates of categorization: P300 amplitude as index of target similarity, Biol Psychol. 71 (2006) 278-288.

3. R.A. Barkley, Behavioral inhibition, sustained attention, and executive functions: Constructing a unifying theory of ADHD, Psychol Bull. 121 (1997) 65-94.

4. R.A. Barkley, K.R. Murphy, Attention-deficit Hyperactivity Disorder: A Clinical Workbook, 2th ed, The Guilford Press, New York, 1998.

5. R.J. Barry, S.J. Johnstone, A.R. Clarke, A review of electrophysiology in attention-deficit/hyperactivity disorder: II. Event related potentials, Clin Neurophysiol. 114 (2003) 184-198.

6. E. Courchesne, S.A. Hillyard, R. Galambos, Stimulus novelty, task relevance and the visual evoked potential in man, Electroencephalogr Clin Neurophysiol. 39 (1975) 131-143.

7. S. Durston, N.T. Tottenham, K.M. Thomas, M.C. Davidson, I.M. Eigsti, Y. Yang, A.M. Ulug, B.J. Casy, Differential patterns of striatal activation in young children with and without ADHD, Biol Psychiatry. 53 (2003) 871-878.

8. C. Escera, K. Alho, E. Schröger, I. Winkler, Involuntary attention and distractibility 
as evaluated with event-related brain potentials, Audiol Neurootol. 5 (2000) 151-166.

9. D. Friedman, Y.M. Cycowicz, H. Gaeta, The novelty P3: An event-related brain potential (ERP) sign of the brain's evaluation of novelty, Neurosci Biobehav Rev. 25 (2001) 355-373.

10. T. Frodl, E.M. Meisenzahl, D. Müller, G. Leinsinger, G. Juckel, K. Hahn, H.J. Möller, U. Hegerl, The effect of the skull on event-related P300, Clin Neurophysiol. 112 (2001) 1773-1776.

11. P.J. Holcomb, P.T. Ackerman, R.A. Dykman, Auditory event-related potentials in attention and reading disabled boys, Int J Psychophysiol. 3 (1986) 263-273.

12. J. Intriligator, J. Polich, On the relationship between background EEG and the P300 event-related potential, Biol Psychol. 37 (1994) 207-218.

13. S.J. Johnstone, R.J. Barry, Auditory event-related potentials to a two-tone discrimination paradigm in attention deficit hyperactivity disorder, Psychiatry Res. 64 (1996) 179-192.

14. J. Katayama, J. Polich, P300 from one-, two-, and three-stimulus auditory paradigms, Int J Psychophysiol. 23 (1996) 33-40.

15. J. Katayama, J. Polich, P300, probability, and the three-tone paradigm, Electroencephalogr Clin Neurophysiol. 100 (1996) 555-562.

16. J. Katayama, J. Polich, Stimulus context determines P3a and P3b, 
Psychophysiology 35 (1998) 23-33.

17. J. Katayama, J. Polich, Auditory and visual P300 topography from a 3 stimulus paradigm, Clin Neurophysiol. 110 (1999) 463-468.

18. A. Kok, On the utility of $\mathrm{P} 3$ amplitude as a measure of processing capacity, Psychophysiology 38 (2001) 557-577.

19. M. Kutas, G. McCarthy, E. Donchin, Augmenting mental chronometry: The P300 as a measure of stimulus evaluation time, Science 197 (1977) 792-795.

20. G. McCarthy, E. Donchin, A metric for thought: A comparison of P300 latency and reaction time, Science 211 (1981) 77-80.

21. J.J. McGough, R.A. Barkley, Diagnostic controversies in adult attention deficit hyperactivity disorder, Am J Psychiatry. 161 (2004) 1948-1956.

22. J.T. Nigg, Neuropsychologic theory and findings in attention-deficit/hyperactivity disorder: The state of the field and salient challenges for the coming decade, Biol Psychiatry. 57 (2005) 1424-1435.

23. T.W. Picton, The P300 wave of the human event-related potential, J Clin Neurophysiol. 9 (1992) 456-479.

24. J. Polich, Overview of P3a and P3b. In: J Polich (Ed.), Detection of Change: Event-Related Potential and fMRI Findings, Kluwer Academic Press, Boston, 2003, pp. 83-98.

25. J. Polich, J.R. Criado, Neuropsychology and neuropharmacology of P3a and P3b, 
Int J Psychophysiol. 60 (2006) 172-185.

26. J. Polich, A. Kok, Cognitive and biological determinants of P300: An integrative review, Biol Psychol. 41 (1995) 103-146.

27. J.A. Sergeant, H. Geurts, J. Oosterlaan, How specific is a deficit of executive functioning for Attention-Deficit/Hyperactivity Disorder? Behav Brain Res. 130 (2002) 3-28.

28. K.M. Spencer, J. Dien, E. Donchin, Spatiotemporal analysis of the late ERP responses to deviant stimuli, Psychophysiology 38 (2001) 343-358.

29. S. Sutton, M. Braren, J. Zubin, E.R. John, Evoked-potential correlates of stimulus uncertainty, Science 150 (1965) 1187-1188.

30. M.F. Ward, P.H. Wender, F.W. Reimherr, The Wender Utah Rating Scale: An aid in the retrospective diagnosis of childhood attention deficit hyperactivity disorder, Am J Psychiatry. 150 (1993) 885- 890.

31. T.D. Watson, A. Azizian, S. Berry, N.K. Squires, Event-related potentials as an index of similarity between words and pictures, Psychophysiology 42 (2005) $361-368$.

32. C. Wickens, A. Kramer, L. Vanasse, E. Donchin, Performance of concurrent tasks: A psychophysiological analysis of the reciprocity of information processing resources, Science 221 (1983) 1080-1082.

33. T.R. Wodushek, C.S. Neumann, Inhibitory capacity in adults with symptoms of 
Attention Deficit/Hyperactivity Disorder (ADHD), Arch Clin Neuropsychol. 18 (2003) 317-330. 
Table 1. Mean Reaction Times and Performance Rates for Each Task Condition

Typical

410

67

$S D$

Hit rate $(\%)$

$S D$

Reaction time (ms)

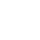

False positive (\%)

Standard

$S D$

Non-target

$S D$
0.1

0.2

0.2

0.3

0.3

0.8

0.8

.2

.3

.8
Novel

410

72

99.5

1.1 


\section{Figure Captions}

Fig. 1. Grand averaged ERPs for each condition from four midline electrode sites $(N$ $=24)$.

Fig. 2. Correlations between the $\mathrm{AD} / \mathrm{HD}$ symptom score and the non-target/target P300 amplitude ratio in each condition. A correlation analysis excluding outlier data $(\mathrm{AD} / \mathrm{HD}$ symptom score $=115)$ also showed a similar result pattern; $r(21)=.64, p<.001$ and $r(21)=.19, p>.3$ for the typical and novel conditions, respectively. 


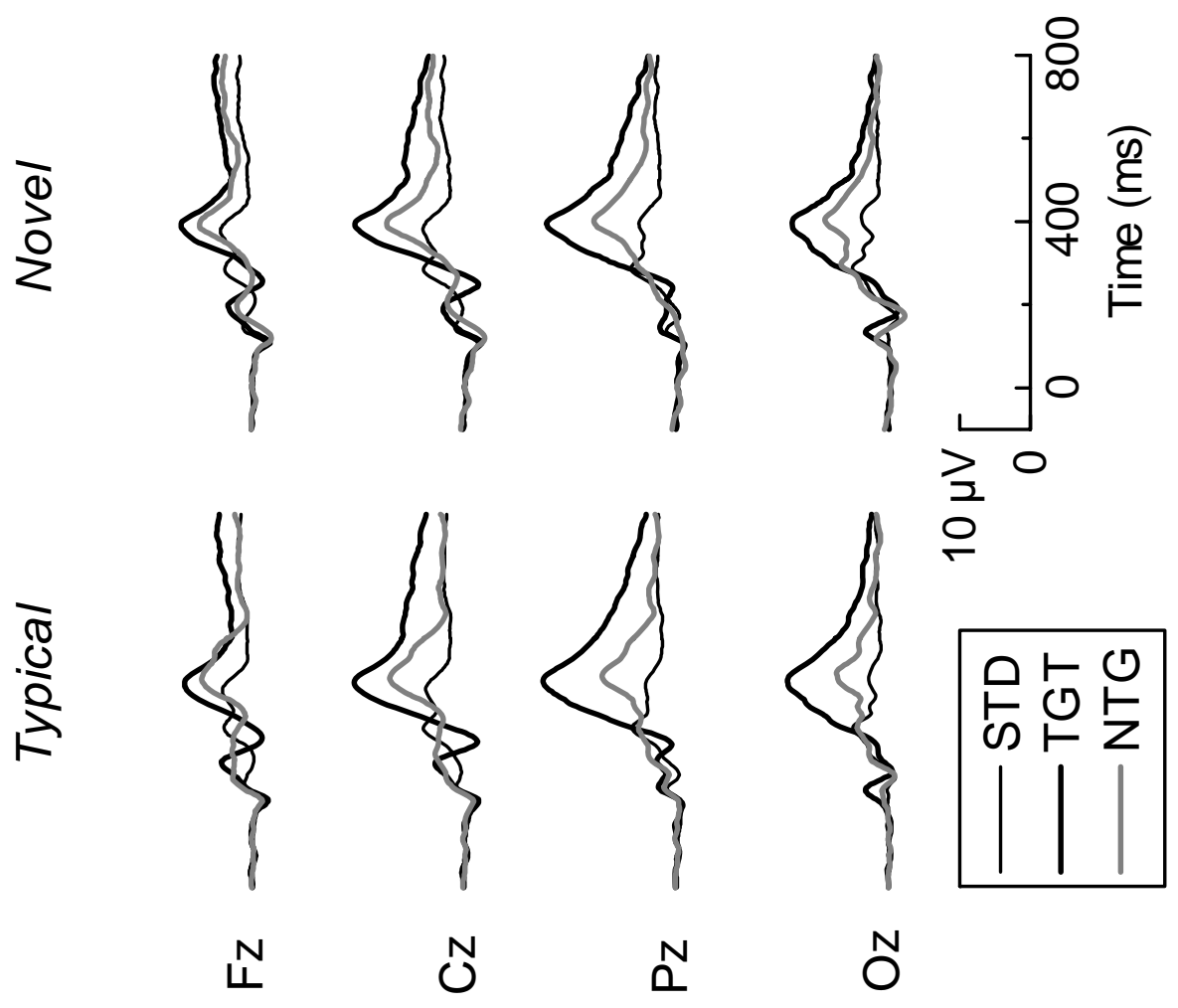

Figure 1. 

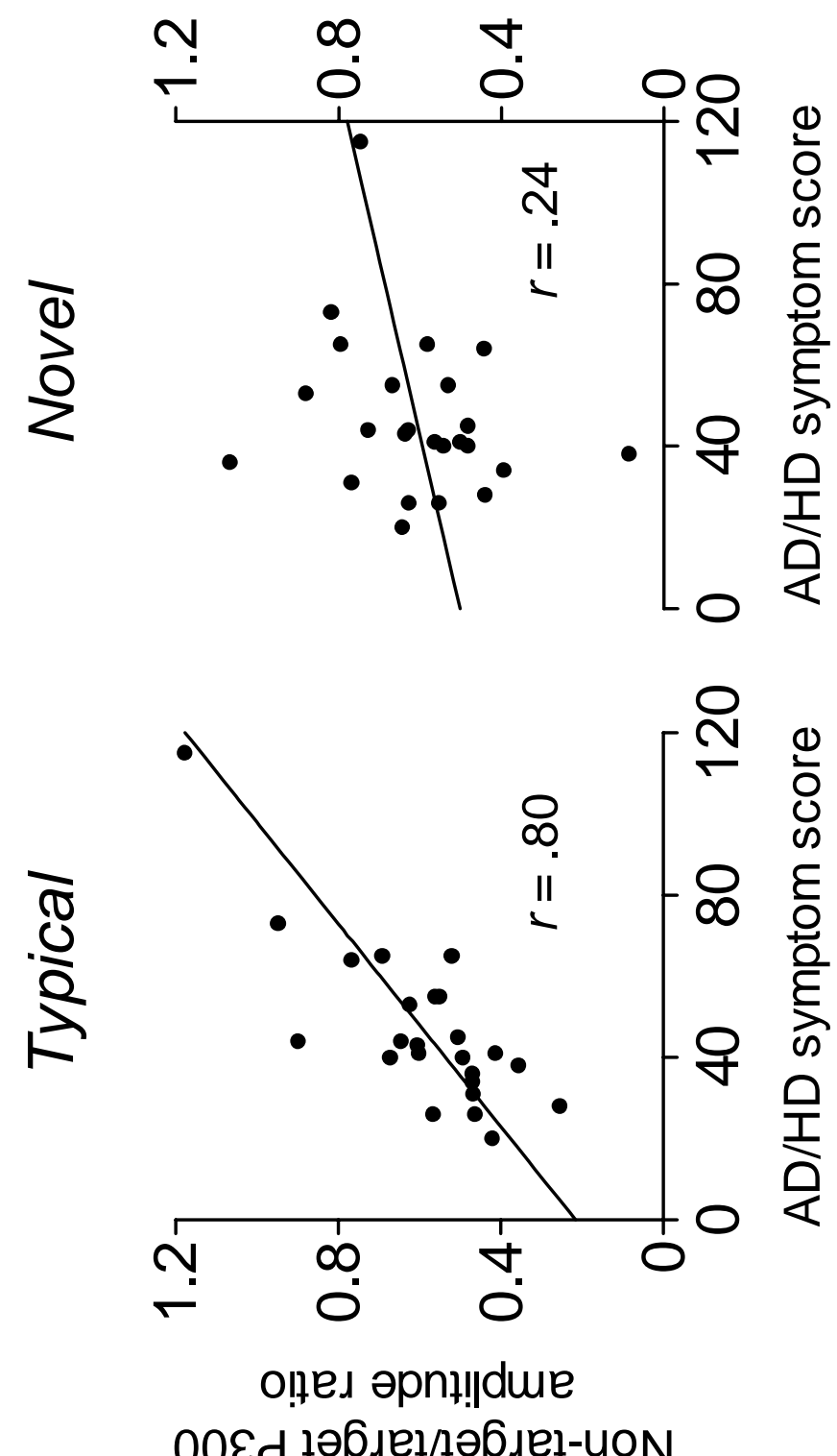

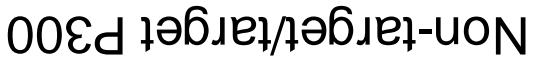

Figure 2. 